Notre Dame Journal of Formal Logic

Volume 40, Number 3, Summer 1999

\title{
On the Consistency Strength of Two Choiceless Cardinal Patterns
}

\author{
ARTHUR W. APTER
}

\begin{abstract}
Using work of Devlin and Schindler in conjunction with work on Prikry forcing in a choiceless context done by the author, we show that two choiceless cardinal patterns have consistency strength of at least one Woodin cardinal.
\end{abstract}

1 Introduction and preliminaries One of the most intriguing problems in large cardinals without the Axiom of Choice is to obtain a model for the theory $T_{1}=$ " $\mathrm{ZF}$ $+\mathrm{DC}_{\kappa}+\kappa$ is a strong limit cardinal + Both $\kappa$ and $\kappa^{+}$are measurable cardinals". It was partially with this motivation that Bull, in his 1976 thesis (published in [6), proved the following theorem.

Theorem 1.1 Con(ZFC $+\kappa<\lambda$ are cardinals so that $\kappa$ is $\lambda$ supercompact and $\lambda$ is measurable $) \Longrightarrow \mathrm{Con}\left(\mathrm{ZF}+D C_{\kappa}+\kappa\right.$ is both a strong limit and Ramsey cardinal $+\kappa^{+}$is measurable).

For the duration of this paper, we will refer to the weakening of $T_{1}$ for which the relative consistency was obtained in Theorem 1.1 as $T_{2}$.

It is unfortunately the case, as Bull showed in [6], that $\kappa$ is not measurable in the model for Theorem 1.1. Nonetheless, Bull's result was the starting point for many investigations into the relative consistency of sequences of consecutive large cardinals, such as those carried out and exposited in Apter [2] and [3], and Apter and Henle [5].

It is interesting to note that Bull's technique and the generalizations thereof given in 2] and [3] require the use of supercompactness. This issue is addressed in greater detail in Apter and Hamkins [4]. This still does not address, however, the exact consistency strength of either of the theories $T_{1}$ or $T_{2}$. Indeed, since neither of the cardinal patterns given by $T_{1}$ and $T_{2}$ follows from $\mathrm{AD}$, and both of these cardinal patterns appear to have consistency strength much stronger than $\mathrm{AD}$, it is quite conceivable that supercompactness will turn out to be necessary in establishing the relative consistency of both $T_{1}$ and $T_{2}$.

Received June 8, 1999; revised July12, 2000 
The purpose of this paper is to show that earlier work on Prikry forcing in a choiceless context given in Apter [1] combined with techniques Devlin uses to prove Theorems 1 and 2 of $[7$ and Schindler uses to prove Theorem 1 of 12 yield, modulo an assumption necessary to carry out certain core model arguments, that the consistency strength of a weakening of $T_{1}$ different from either of the hypotheses used in 12 is at least one Woodin cardinal. Specifically, we prove the following.

Theorem 1.2 Suppose $V \models " Z F+D C+\kappa$ is a limit cardinal + Unboundedly many in $\kappa$ successor cardinals $\delta<\kappa$ are regular $+\kappa$ carries a shrinking filter $+\kappa^{+}$ is measurable $+\Omega>\kappa^{+}$is inaccessible and $V_{\Omega}^{\mathrm{HOD}}$ is closed under sharps". There is then an inner model with one Woodin cardinal.

As an immediate corollary to Theorem 1.2. we obtain the following theorem.

Theorem 1.3 Suppose $V \models " Z F+\kappa$ is a limit cardinal + Unboundedly many in $\kappa$ successor cardinals $\delta<\kappa$ are regular $+\kappa$ carries a shrinking filter $+\kappa^{+}$is singular $+\Omega>\kappa^{+}$is inaccessible and $V_{\Omega}^{\mathrm{HOD}}$ is closed under sharps". There is then an inner model with one Woodin cardinal.

The referee has pointed out that it is possible to weaken the hypotheses of Theorem 1.2 to " $\mathrm{ZF}+\kappa$ is a limit cardinal + Unboundedly many in $\kappa$ successor cardinals $\delta<\kappa$ are regular $+\kappa$ carries a shrinking filter $+\kappa^{+}$is weakly compact $+\Omega>\kappa^{+}$ is inaccessible and $V_{\Omega}^{\mathrm{HOD}}$ is closed under sharps". We will provide details of the referee's proof in Section 3 .

The terminology we use in Theorems 1.2 and 1.3 s that given in [7] and [12]. Thus, as in 12], in a choiceless context, a cardinal $\delta$ is inaccessible if and only if for no cardinal $\alpha<\delta$ does a cofinal map $f: V_{\alpha} \rightarrow \delta$ exist. As in [7], the cardinal $\delta$ carries a shrinking filter $\mathcal{D}$ if and only if whenever $A \in \mathcal{D}$ and $f:[A]^{<\omega} \rightarrow \alpha^{+}<\delta$, there is $B \subseteq A, B \in \mathcal{D}$ so that $\left|f^{\prime \prime}[B]^{<\omega}\right| \leq \alpha$. Rowbottom's theorem implies that if $\delta>\aleph_{1}$ is a measurable cardinal carrying a normal measure, then $\delta$ carries a shrinking filter, that is, the normal measure itself. However, as shown by Prikry in [11, it is possible for $\delta>\aleph_{1}$ to carry a shrinking filter and be singular.

To prove Theorems 1.2 and 1.3. we will need the following two lemmas. Lemma 1.4 appears as Lemma 3 of [12 and is a generalization of a theorem of Vopěnka. (Vopěnka's original theorem can be found as Theorem 65, pp. 293-94 of Jech [8].) Lemma 1.5 is a slight strengthening of Lemma 4 of [12]. The results given in the first and second sentences of the conclusions of Lemma 1.5 are due to Steel and can be found in Schimmerling [13] and Steel [14]. The results given in the third and fourth sentences of the conclusions of Lemma 1.5 are due to Mitchell, Schimmerling, and Steel and can be found in [10] and 92 .

Lemma 1.4 Let $x \subseteq \operatorname{HOD}[a] \subseteq V$ for some $a$ and $x$. There is then a partial ordering $\mathbb{P} \in \operatorname{HOD}[a]$ so that $x$ is $\operatorname{HOD}[a]$-generic over $\mathbb{P}$. Further, if $x \subseteq V_{\alpha}^{\mathrm{HOD}[a]}$ for some $\alpha<\delta$, where $V \models$ " $\delta$ is inaccessible", then $\mathbb{P}$ can be chosen so that $\mathbb{P} \in V_{\delta}^{\mathrm{HOD}[\mathrm{a}]}$.

Lemma 1.5 Let $N \subseteq V, N \models \mathrm{ZFC}$ be an inner model of $V$ so that $N \models$ " $\delta$ is inaccessible and $V_{\delta}$ is closed under sharps". Suppose there is no inner model of $V$ with a Woodin cardinal. Then the core model $K=K^{N}$ inside $N$ up to $\delta$ exists and is $\delta$ iterable. Also, if $M=N^{\mathbb{P}}$ for some $\mathbb{P} \in V_{\delta}^{N}$, then $K^{M}=K$. Further, if $N \models " \aleph_{2} \leq \alpha<\delta$ 
is a cardinal", then $N \models$ " $\operatorname{cof}\left(\left(\alpha^{+}\right)^{K}\right) \geq \alpha$ ". In particular, if $N \models$ " $\aleph_{2} \leq \alpha<\delta$ is a singular cardinal", then $N \models$ “ $\left(\alpha^{+}\right)^{K}=\alpha^{+}$".

2 The proofs of Theorems 1.2 and 1.3 We turn now to the proof of Theorem 1.2 .

Proof: We assume $V$ is as in the hypotheses of Theorem 1.2. Since $V \models$ "ZF + $\mathrm{DC}+\kappa^{+}$is measurable", we know that $\kappa^{+}$carries a normal measure. Thus, by the work of Section 1 of [1], it is possible to define a Prikry partial ordering $\mathbb{P}_{1} \in V$ so that $V_{1}=V^{\mathbb{P}_{1}} \models$ "The bounded subsets of $\kappa^{+}$are the same as in $V+\kappa^{+}$is a singular cardinal of cofinality $\omega$ ". Hence, $\left(\kappa^{+}\right)^{V_{1}}=\left(\kappa^{+}\right)^{V}$, and since by hypothesis $V \models$ " $\kappa$ carries a shrinking filter $\mathcal{D}$ ", $V_{1}=$ " $\mathcal{D}$ is a shrinking filter" as well.

Let $\mathbb{P}_{0} \in V_{1}$ be Prikry forcing over $\kappa$ defined using $\mathcal{D}$. By Lemma 1.3 of [1], which does not require that $\mathcal{D}$ be a normal measure, $V_{1}^{\mathbb{P}_{0}}=V_{2} \models$ " $\kappa^{+}=\left(\kappa^{+}\right)^{V_{1}}$ and hence is a singular cardinal (of cofinality $\omega$ )".

We show now that $V_{2}=$ " $\kappa$ is a singular cardinal of cofinality $\omega$ ". Clearly, the standard density arguments yield $V_{2}=$ " $\operatorname{cof}(\kappa)=\omega$ ", so it suffices to prove $V_{2}=$ " $\kappa$ is a cardinal". We do this by using the argument Devlin employs in the proof of Theorem 1 of [7]. Specifically, we show forcing with $\mathbb{P}_{0}$ preserves that every regular successor cardinal below $\kappa$ is a cardinal. Since $V_{1} \models$ " $\kappa$ is a limit cardinal and unboundedly many in $\kappa$ successor cardinals $\delta<\kappa$ are regular", this will yield that forcing with $\mathbb{P}_{0}$ over $V_{1}$ preserves that $\kappa$ is a cardinal. Therefore, if $\delta=\lambda^{+}<\kappa$ is the least $V_{1}$-regular successor cardinal so that for some $p=\left\langle s_{0}, S_{0}\right\rangle \in \mathbb{P}_{0}, p \Vdash$ " $\delta$ isn't a cardinal", then we can assume without loss of generality that $p \Vdash$ " $\dot{f}: \lambda \rightarrow \delta$ is a surjection".

For each $s \in\left[S_{0}\right]^{<\omega}$, let $T_{s}=\left\{\beta<\delta\right.$ : for some condition $q=\left\langle s_{0} \frown s, A\right\rangle$ extending $p$ and some $\alpha<\lambda, q \Vdash$ " $\dot{f}(\alpha)=\beta$ " $\}$. Note that if $\langle t, A\rangle,\langle t, B\rangle \in \mathbb{P}_{0},\langle t, A \cap B\rangle$ extends both of these conditions. Thus, $T_{s}$ is well defined and has cardinality at most $\lambda$. Therefore, by our hypothesis that $\delta$ is regular in $V_{1}, F:\left[S_{0}\right]^{<\omega} \rightarrow \delta$ given by $F(s)=\sup \left(T_{s}\right)$ is such that $F(s)$ defines an ordinal below $\delta$. Since $\mathcal{D}$ is a shrinking filter in both $V$ and $V_{1}$, this means that for some $S_{1} \subseteq S_{0}, S_{1} \in \mathcal{D},\left|F^{\prime \prime}\left[S_{1}\right]^{<\omega}\right| \leq \lambda$ and $\gamma=\sup \left(F^{\prime \prime}\left[S_{1}\right]^{<\omega}\right)<\delta$. However, $p^{\prime}=\left\langle s, S_{1}\right\rangle$ extends $p$ and $p^{\prime} \Vdash " \dot{f}^{\prime \prime} \lambda \subseteq \gamma+1<$ $\delta^{\prime \prime}$, a contradiction. Thus, all regular successor cardinals $\delta<\kappa$ in $V$ and $V_{1}$ remain cardinals in $V_{2}$.

We continue by using a modification of the argument employed by Schindler in the proof of Theorem 1 of [12]. We assume that there is no inner model of $V_{2}$ with a Woodin cardinal, which immediately implies that there is no inner model of either $V_{1}$ or $V$ with a Woodin cardinal. Let $t_{1}$ and $t_{2}$ be the cofinal $\omega$ sequences present in $V_{2}$ through $\kappa$ and $\kappa^{+}$respectively, and let $M=\mathrm{HOD}^{V_{2}}$.

Note that $\Omega$ remains inaccessible in $V_{2}$. This is since $\mathbb{P}_{1} * \mathbb{P}_{0} \in V_{\Omega}$ and every $x \in V_{\Omega}^{V_{2}}$ has a name $\dot{x} \in V_{\Omega}$ which then implies that there are no $p \in \mathbb{P}_{1} * \mathbb{P}_{0}, \tau$, and $\alpha<\Omega$ with $p \Vdash$ " $\tau: V_{\alpha}^{V_{2}} \rightarrow \Omega$ is a cofinal map". Further, by the homogeneity of $\mathbb{P}_{1} * \mathbb{P}_{0}, M$ is a definable inner model of $V$, since a set $x$ of ordinals is ordinal definable in $V_{2}$ if and only if there is a formula $\varphi$ and an ordinal sequence $\vec{\alpha}$ such that $\xi \in x \Longleftrightarrow$ $\vdash_{\mathbb{P}_{1} * \mathbb{P}_{0}} \varphi(\xi, \vec{\alpha})$.

Assume that $x \subseteq \Omega$ is bounded and ordinal definable in $V_{2}$. Work for the time being in $V$. By the preceding paragraph, $x \in V_{\Omega}$, so by Lemma 1.4, $x \in \mathrm{HOD}^{\mathbb{Q}}$ for 
some $\mathbb{Q} \in V_{\Omega}^{\mathrm{HOD}}$. Thus, since the $V_{\Omega}$ of every small generic extension of HOD is closed under sharps, we are now able to infer that $V_{2} \models$ " $\Omega$ is inaccessible and $V_{\Omega}^{\mathrm{HOD}}$ (as computed in $V_{2}$ ) is closed under sharps". Therefore, $M \models$ " $\Omega$ is inaccessible and $V_{\Omega}$ is closed under sharps", so by Lemma $1.5, K=K^{M}$ inside $M$ up to $\Omega$ exists and is $\Omega$ iterable in $M$.

We claim now that $\left(\kappa^{+}\right)^{K}=\left(\kappa^{+}\right)^{V}=\left(\kappa^{+}\right)^{V_{1}}=\left(\kappa^{+}\right)^{V_{2}}$. If not, then let $f: \kappa \rightarrow$ $\left(\kappa^{+}\right)^{K}, f \in V_{2}$ be a bijection. By Lemma 1.4 $M\left[\left\langle t_{1}, f\right\rangle\right]$ is a generic extension of $M$ via a partial ordering which is an element of $V_{\Omega}^{M}$. Hence, Lemma 1.5 mplies that inside $M\left[\left\langle t_{1}, f\right\rangle\right]$ and up to $\Omega, K=K^{M}=K^{M\left[\left\langle t_{1}, f\right\rangle\right]}$. Since $\kappa$ is a singular cardinal in $M\left[\left\langle t_{1}, f\right\rangle\right]$, another application of Lemma 1.5 hen yields that $\left(\kappa^{+}\right)^{K}=\left(\kappa^{+}\right)^{M\left[\left\langle t_{1}, f\right\rangle\right]}$. On the other hand, it is, of course, the case that $M\left[\left\langle t_{1}, f\right\rangle\right] \models$ " $\left(\kappa^{+}\right)^{K}<\kappa^{+}$". From this contradiction, we therefore know that $\left(\kappa^{+}\right)^{K}=\kappa^{+}$, and so $M\left[\left\langle t_{2}, t_{1}\right\rangle\right] \models$ "ZFC $+\left(\kappa^{+}\right)^{K}=\kappa^{+}+\kappa^{+}$is a singular cardinal". This final contradiction proves Theorem 1.2 .

As indicated in Section 1. Theorem 1.3 is an immediate corollary of Theorem 1.2. Instead of choosing $t_{2}$ as being Prikry generic for the measurable cardinal $\kappa^{+}$, one simply chooses $t_{2}$ as being a cofinal sequence through $\kappa^{+}$witnessing that $\kappa^{+}$is singular. The remaining details of the proof of Theorem 1.3 are then virtually identical to the proof of Theorem 1.2. as readers can easily verify for themselves.

3 Concluding remarks In conclusion, we remark that in the first version of this paper, we asked if the hypotheses of Theorem 1.2 could be weakened so that the assumptions of DC were dropped and $\kappa^{+}$were just assumed to be weakly compact and not measurable. As we mentioned in Section 1 the referee has provided a proof that this is indeed possible. Here are the details: Let $\mathbb{P}_{0}$ be as in the proof of Theorem 1.2. Force with $\mathbb{P}_{0}$ over $V$. By the arguments given in the proof of Theorem $1,2, K$ can be constructed in $\mathrm{HOD}^{V^{\mathbb{P}_{0}}}$, and we will as before have that $\left(\kappa^{+}\right)^{K}=\left(\kappa^{+}\right)^{V}=\left(\kappa^{+}\right)^{V^{\mathbb{P}_{0}}}$. Thus, $\left(\kappa^{+}\right)^{V}$ is a successor cardinal in $K$. On the other hand, by the local definability of $K$ (see [14], §6) and the homogeneity of $\mathbb{P}_{0}$, it will be the case that $x$ is a set of ordinals in $K$ if and only if there is a formula $\varphi$ and an ordinal sequence $\vec{\alpha}$ such that $\xi \in x \Longleftrightarrow \Vdash_{\mathbb{P}_{0}} \varphi(\xi, \vec{\alpha})$. This implies that $K$ is a definable inner model of $V$. This means we can then use Lemma 1(d) of [12] to infer that $\left(\kappa^{+}\right)^{V}$, which by hypothesis is weakly compact, is inaccessible in $K$. This contradiction proves the improved version of Theorem 1.2 .

Acknowledgments The author owes a huge debt of gratitude to the referee, whom he wishes to thank for patience shown toward the author's questions, for thoroughly reading the original manuscript and pointing out numerous errors and suggesting numerous improvements, and for providing the proof for the improved form of Theorem 1.2 given at the end of the paper. The referee's influence is omnipresent in this version of the paper.

\section{REFERENCES}

[1] Apter, A., "AD and patterns of singular cardinals below $\Theta$," The Journal of Symbolic Logic, vol. 61 (1996), pp. 225-35.Zbl 0855.03029|MR 97d:03071 1.12.12

[2] Apter, A., "Some results on consecutive large cardinals," Annals of Pure and Applied Logic, vol. 25 (1983), pp. 1-17. Zbl 0548.03030|MR 85f:03053 1. ID 
[3] Apter, A., "Some results on consecutive large cardinals II: Applications of Radin forcing," Israel Journal of Mathematics, vol. 52 (1985), pp. 273-92.

Zbl 0603.03016|MR 87j:03073 1,1.1

[4] Apter, A., and J. D. Hamkins, "Indestructible weakly compact cardinals and the necessity of supercompactness for certain proof schemata," Mathematical Logic Quarterly, vol. 47 (2001), pp. 563-71.

[5] Apter, A., and J. Henle, "Large cardinal structures below $\aleph_{\omega}$," The Journal of Symbolic Logic, vol. 51 (1986), pp. 591-603.Zbl 0634.03050|MR 87k:03054 1

[6] Bull, E., "Consecutive large cardinals," Annals of Mathematical Logic, vol. 15 (1978), pp. 161-91. 1,1

[7] Devlin, K., "Some remarks on changing cofinalities," The Journal of Symbolic Logic, vol. 39 (1974), pp. 27-30.Zbl 0286.02072MR 50:109 1. 1.11.12

[8] Jech, T., Set Theory, Academic Press, New York, 1978. Zbl 0419.03028||MR 80a:03062 1

[9] Mitchell, W., and E. Schimmerling, "Covering without countable closure," Mathematical Research Letters, vol. 2 (1995), pp. 595-609.

Zbl 0847.03024||MR 96k:03123 1

[10] Mitchell, W., E. Schimmerling, and J. Steel, "The covering lemma up to a Woodin cardinal," Annals of Pure and Applied Logic, vol. 84 (1997), pp. 219-55. Zbl 0868.03021||MR 98b:030671

[11] Prikry, K., "Changing measurable into accessible cardinals," Dissertationes Mathematicae, vol. 68 (1970), pp. 5-52.Zbl 0212.32404|MR 41:6685 1

[12] Schindler, R., "Successive weakly compact or singular cardinals," The Journal of Symbolic Logic, vol. 64 (1999), pp. 139-46. Zbl 0926.03069||MR 2000f:03152 1, 1, 1, 1, 1, 1, 1, 1, |2, ,3

[13] Schimmerling, E., "Combinatorial principles in the core model for one Woodin cardinal," Annals of Pure and Applied Logic, vol. 74 (1995), pp. 153-201. Zbl 0834.03018|MR 96f:03041 1

[14] Steel, J., The Core Model Iterability Problem, Lecture Notes in Logic 8, SpringerVerlag, Berlin, 1996. Zbl 0864.03035 MR 99k:03043 1.3

Department of Mathematics

Baruch College of CUNY

New York NY 10010

email: awabb@cunvvm.cunv.edu

website: http://math.baruch.cuny.edu/ apter 